\title{
Bindweeds or random walks in random environments on multiplexed trees and their asympotics
}

\author{
Mikhail Menshikov ${ }^{1}$ and Dimitri Petritis ${ }^{2}$ and Serguei Popov ${ }^{3 \dagger}$ \\ ${ }^{1}$ Department of Mathematical Sciences, University of Durham, South Road, Durham DH1 3LE, United Kingdom \\ Mikhail.Menshikov@durham.ac.uk \\ ${ }^{2}$ Institut de Recherche Mathématique, Université de Rennes I and CNRS UMR 6625, 35042 Rennes Cedex, France \\ Dimitri.Petritiseuniv-rennes1.fr \\ ${ }^{3}$ Instituto de Matemática e Estatística, Universidade de São Paulo, Rua do Matão 1010, CEP 05508-090, São Paulo \\ SP, Brasil \\ popoveime.usp.br
}

\begin{abstract}
We report on the asymptotic behaviour of a new model of random walk, we term the bindweed model, evolving in a random environment on an infinite multiplexed tree. The term multiplexed means that the model can be viewed as a nearest neighbours random walk on a tree whose vertices carry an internal degree of freedom from the finite set $\{1, \ldots, d\}$, for some integer $d$. The consequence of the internal degree of freedom is an enhancement of the tree graph structure induced by the replacement of ordinary edges by multi-edges, indexed by the set $\{1, \ldots, d\} \times\{1, \ldots, d\}$. This indexing conveys the information on the internal degree of freedom of the vertices contiguous to each edge. The term random environment means that the jumping rates for the random walk are a family of edge-indexed random variables, independent of the natural filtration generated by the random variables entering in the definition of the random walk; their joint distribution depends on the index of each component of the multi-edges. We study the large time asymptotic behaviour of this random walk and classify it with respect to positive recurrence or transience in terms of a specific parameter of the probability distribution of the jump rates. This classifying parameter is shown to coincide with the critical value of a matrix-valued multiplicative cascade on the ordinary tree (i.e. the one without internal degrees of freedom attached to the vertices) having the same vertex set as the state space of the random walk. Only results are presented here since the detailed proofs will appear elsewhere.
\end{abstract}

Keywords: Markov chain, trees, random environment, recurrence criteria, matrix multiplicative cascades

\section{Introduction}

\subsection{On the generality of the random walk in a random environment on a tree}

Markov chains on denumerable graphs enter in the modeling of a rich variety of phenomena; among such graphs, trees play a basic and generic rôle in the sense that they can encode simultaneously

\footnotetext{
${ }^{\dagger}$ Partially supported by CNPq (302981/02-0)

1365-8050 @ 2003 Discrete Mathematics and Theoretical Computer Science (DMTCS), Nancy, France
} 
- the topological structure of a vast class of general (i.e. not necessarily tree-like) denumerable graphs,

- the combinatorial structure of paths on these general graphs, and

- the probability structure generated on the trajectory space of the Markov chain evolving on the these general graphs.

In the sequel we give a short explanation of the reason trees play such a generic rôle among denumerable graphs. We know, after Kolmogorov, that in order to describe a random variable $X$ with space of outcomes a discrete measurable set $(\mathbb{X}, X)$, of law $\mathbb{P}_{X}$, one has to use an abstract probability space $(\Omega, \mathcal{F}, \mathbb{P})$ on which the random variable is defi ned. However, the choice of this space is not unique. Among the infi nite possible choices, there exists a "minimal" one, given by $\Omega=\mathbb{X}$, realizing the random variable as the identity map $X(\omega)=\omega$ and $\mathbb{P}=\mathbb{P}_{X}$. Similarly, when $\left(X_{n}\right)_{n \in \mathbb{N}}$ is a sequence of independent and identically distributed random variables with space of outcomes the discrete space $(\mathbb{X}, X)$, the minimal realization of the abstract probability space carrying the whole sequence is the space of trajectories or full shift $\Omega=\mathbb{X}^{\mathbb{N}}$ and the sequence is realized by the canonical projection $X_{n}(\omega)=\omega_{n}$, for $n \in \mathbb{N}$. However for a sequence of dependent random variables the space of trajectories may be not minimal. The reader can easily convince herself by considering the example of $\mathbb{X}=\{a, b, \ldots, z\}$ and $\left(X_{n}\right)_{n \in \mathbb{N}}$ being the sequence of letters appearing in a natural language. Then the occurrence $\left(X_{n}, X_{n+1}, X_{n+2}\right)=r z t$ never appears in a language like English or French.

For the special case of Markovian dependence, the natural space for the realization of the sequence $\left.\left(X_{n}\right)_{n \in \mathbb{N}}\right)$ is the so called unilateral or bilateral subshift spaces obtained from the full shift by deleting all sequences containing forbidden subwords. For Markovian sequences, defi ned through a stochastic matrix $P$, subshift spaces can also be obtained in terms of the adjacency matrix of the sequence $A: \mathbb{X} \times \mathbb{X} \rightarrow$ $\{0,1\}$, given by the formula $A(x, y)=1$ whenever $P(x, y)>0$ and zero otherwise.

Suppose now we are given an arbitrary directed graph having a fi nite or denumerable set of vertices and being such that only a fi nite number of edges is emitted out of every vertex, not having any sources or sinks, and having (complex) weights attached on its edges. Since the vertex set is at most countable, it is in bijection with an at most countable alphabet. We say that a sequence of letters from the alphabet is a path of the graph, if the corresponding sequence of vertices is such that any ordered pair of subsequent vertices is an element of the edge set. The set of paths of arbitrary length is called path space of the graph (see [13] for precise defi nitions.) Notice that the condition that two subsequent vertices must be an allowed edge prevents some sequences of vertices from being a path. The adjacency matrix defi nes a subshift space for the trajectories of a symbolic unilateral or bilateral Markov chain that is identifi ed with the path space of the graph. It is easy to show that the path space of any graph has a natural tree structure [1], giving thus a fi rst hint that trees play a prominent rôle among graphs. Their importance does not stop here however. We can in fact defi ne a so-called evaluation map from the path space of a graph into some set $\mathbb{A}$. Depending on the precise algebraic structure of the set $\mathbb{A}$ and of the evaluation map, a vast class of objects can be defi ned. Instead of giving precise defi nitions, let us give the following

Example 1.1 (An elementary case) Start with the fi nite complete graph on 4 vertices, denote them by the letters $\mathrm{E}, \mathrm{N}, \mathrm{W}$, and $\mathrm{S}$ for defi niteness, and consider the unilateral path space of the graph, i.e. the set of words of arbitrary length on the alphabet of these 4 letters. Choose for the space $\mathbb{A}$ the Abelian group $\mathbb{Z}^{2}$ and for the evaluation map the function taking the value $n_{E} \mathbf{e}_{1}+n_{N} \mathbf{e}_{2}+n_{W}\left(-\mathbf{e}_{1}\right)+n_{S}\left(-\mathbf{e}_{2}\right) \in \mathbb{Z}^{2}$, where $n_{E}, n_{N}, n_{W}, n_{S}$ denote the occurrences of the letters $\mathrm{E}, \mathrm{N}, \mathrm{W}$, and $\mathrm{S}$ in a given word, and $\mathbf{e}_{1}, \mathbf{e}_{2}$ are 
the unit vectors of $\mathbb{Z}^{2}$. The evaluation map can be thought as the physical observable "position of the random walker" when the history of the individual directions of movement is kept in memory; individual directions defi ne a path in the path space and the evaluation map computes the actual position of the walker which has done $n_{E}$ eastward movements, $n_{W}$ westward movements, etc. The graph that is generated in this way is the graph that coincides with the Cayley graph of the Abelian group $\mathbb{Z}^{2}$. The evaluation map being many-to-one, its multiplicity encodes the combinatorics of the path space while the product of the graph weights gives the relative weight of individual paths in the path space of this graph. When the weights are probability vectors, this weight coincides with the probability of the trajectory of the simple random walk on $\mathbb{Z}^{2}$. Notice however that the weights need not to be probability vectors, allowing us to consider both random and quantum grammars.

The above example serves as a basic paradigm; by appropriately changing the evaluation map, it can be generalised in numerous ways to produce confi guration space for DNA strands (when the path space coincides with the path space of the complete graph over 4 letters), non-Abelian groups like the free group on 2 generators, Cuntz-Krieger $C^{*}$-algebras (i.e non-commutative operator algebras attaching non-zero partial isometries on every edge; see [24] for additional details) naturally arising in some problems of quantum information. Infi nite trees provide a rich variety of mathematical problems, particularly connected to their non-amenability; beyond their mathematical interest they arise as more or less realistic models in several applied fi elds like random search algorithms in large data structures, Internet traffi c, random grammars and probabilistic Turing machines, DNA coding, interacting random strings and automated languages, etc. The previously exposed ideas, already lurking in $[17,19]$, have been exemplifi ed in $[8,1]$, and will be further exploited in [24].

Random environment is a means to introduce context-dependence in the language. Again, due to the generality of trees as underlying graphs, random walks in random environment on trees provide interesting context-dependent results for random walks on a huge class of more general graphs.

Random walks in random environments on various types of graphs are known to display a behaviour dramatically differing from the one for ordinary random walks on the same graph. See $[27,26,11,12,16$, $4,18,22,5,2]$ etc. for a very partial list of known results and models.

\subsection{Rough statement of the main results for bindweeds}

As a simple example, consider a rooted tree with constant branching $b$, and let us construct a random walk in random environment on it by sampling the transition probabilities (or transition rates, for continuous time) in each vertex from a given distribution, independently. When studying the question of (positive) recurrence of such a walk, one naturally arrives (see [16, 22]) at the following model. On each edge $a$ of the tree, we place an independent copy of a positive random variable $\hat{\xi}_{a}$. Then, for each vertex $\mathbf{v}$ denote $\hat{\xi}[\mathbf{v}]=\hat{\xi}_{a_{1}} \ldots \hat{\xi}_{a_{n}}$, where $a_{1}, \ldots, a_{n}$ is the (unique) path connecting the root to $\mathbf{v}$. Models of this type are called multiplicative cascades (see e.g. [10,15]), and, to study the positive recurrence of the corresponding random walk in random environment, one has to answer the question whether the sum of $\hat{\xi}[\mathbf{v}]$ is fi nite. It turns out (see e.g. [16]) that the classifi cation parameter for this problem is

$$
\hat{\lambda}=\inf _{s \in[0,1]} \mathbb{E} \hat{\xi}_{a}^{s},
$$

which is then compared to $1 / b$ (in fact, in [16] the case of general tree was considered) and the following result is established: 
- if $\hat{\lambda} b<1$ then the random walk for almost all environments is positive recurrent

- if $\hat{\lambda} b>1$ then the random walk for almost all environments is transient.

The critical case $\hat{\lambda} b=1$ is more complicated.

In [23], we introduced a model of random walk with internal degrees of freedom whose multiplicative cascade counterpart is expressed by a model placing random matrices on the edges in place of scalar random variables. The main classifi cation parameter, $\lambda$, will be defi ned in the formula (3) below, and the main results are Theorems 3.1 and 3.2 (a lot of preliminary work is required, however, before formulating these results). This model is also equivalent to random walk in random environment on a multiplexed tree, a process we term bindweed in the sequel. However, for trees with average branching $b$, the rough statement of our result is as above with $\hat{\lambda}$ replaced by $\lambda$.

It was remarked in [22] that asymptotic properties like recurrence/transience of random walk on trees with constant branching $b$ are intimately connected to the existence of non-trivial solutions for the socalled multiplicative chaos equation of order $b$, fi rst introduced as a simple turbulence model in [20]. The simplest variant of the multiplicative chaos equation is the following: let $\left(\xi_{i}\right)_{i=1, \ldots, b}$, with $b \in \mathbb{N}$, be a fi nite family of non-negative random variables having known joint distribution and $\left(Y_{i}^{\prime}\right)_{i=1, \ldots, b}$ and $Y$ be a family of $b+1$ independent non-negative random variables distributed according the same unknown law and verifying

$$
Y \stackrel{\operatorname{law}}{=} \sum_{i=1}^{b} Y_{i}^{\prime} \xi_{i}
$$

The multiplicative chaos problem consists in determining under which conditions on the joint distribution of the $\xi$ 's the above equation has a non-trivial solution. This scalar problem is thoroughly studied in the literature, see e.g. $[3,6,14]$. As we remark later in this paper, the matrix multiplicative chaos equation may be an interesting problem to study as well.

\section{Notation}

In this section we give the formal defi nitions concerning trees, in particular, we defi ne the notions of the growth rate and the branching number.

We denote $\mathbb{R}_{+}=\left[0, \infty\left[, \mathbb{Z}_{+}=\{0,1,2, \ldots\}, \mathbb{N}=\{1,2,3, \ldots\}\right.\right.$, and for every $n \in \mathbb{N}, \mathbb{N}_{n}=\{1,2, \ldots, n\}$, while $\mathbb{N}_{0}=\emptyset$. Let $\mathcal{A} \equiv \mathscr{A}^{1}$ be a fi nite or infi nite denumerable set, called the alphabet. Defi ne $\mathscr{A}=\{\emptyset\}$ and for every $n \in \mathbb{N}$ denote

$$
\mathcal{A}^{n}=\left\{\alpha=\alpha_{1} \cdots \alpha_{n}: \alpha_{i} \in \mathcal{A} \text { for } i \in \mathbb{N}_{n}\right\}
$$

the set of words of length $n$ (i.e. having $n$ letters),

$$
\mathcal{A}^{*}=\cup_{n \in \mathbb{Z}_{+}} \mathcal{A}^{n}
$$

the set of words of arbitrary (fi nite) length, and

$$
\partial \mathcal{A}^{*} \equiv \mathcal{A}^{\infty}=\left\{\alpha=\alpha_{1} \alpha_{2} \cdots: \alpha_{i} \in \mathcal{A} \text { for } i \in \mathbb{N}\right\}
$$

the set of infi nite words. Finally, denote $\overline{\mathcal{A}^{*}}=\mathscr{A}^{*} \cup \partial \mathcal{A}^{*}$ and $\stackrel{\circ}{\mathcal{A}^{*}}=\mathcal{A}^{*} \backslash \mathcal{A}^{0}$.

For every $\alpha \in \mathcal{A}^{*}$, there exists $n \in \mathbb{Z}_{+}$such that $\alpha \in \mathcal{A}^{n}$; in this situation $|\alpha|:=n$ denotes the length of the word $\alpha$ with the convention $|\emptyset|=0$. Consistently, for every $\alpha \in \partial \mathcal{A}^{*}$, we have $|\alpha|=\infty$. For $\alpha \in \overline{\mathcal{A}^{*}}$ 
with $|\alpha| \geq n$ we denote by $\alpha \Gamma_{n}=\alpha_{1} \cdots \alpha_{n} \in \mathcal{A}^{n}$ the restriction of $\alpha$ to its $n$ fi rst letters with the convention $\alpha \uparrow_{0}=\emptyset$. For every $\alpha \in \stackrel{\circ}{\mathcal{A}}^{*}$, the ancestor $\hat{\alpha}$ of $\alpha$ is defi ned by $\hat{\alpha}=\alpha \uparrow_{|\alpha|-1}$. For $\alpha \in \mathcal{A}^{*}$ and $\beta \in \overline{\mathcal{A}^{*}}$, the concatenation of $\alpha$ followed by $\beta$ is the word $\alpha \beta=\alpha_{1} \cdots \alpha_{|\alpha|} \beta_{1} \beta_{2} \cdots$ and for $\alpha, \beta \in \mathcal{A}^{*}$, their common radix $\alpha \wedge \beta$ is the longest word $\gamma \in \mathcal{A}^{*}$ such that $\alpha=\gamma \alpha^{\prime}$ and $\beta=\gamma \beta^{\prime}$ for some words $\alpha^{\prime}, \beta^{\prime} \in \mathcal{A}^{*}$. We write $\alpha \leq \beta$ if $\alpha=\alpha \wedge \beta$.

Remark: Notice that, consistently with the above notation, the symbol $\mathbb{N}^{*}$ denotes the set of fi nite words on the alphabet $\mathbb{N}$, contrary to some tradition (especially the French one) where this symbol is used to denote what we call here $\mathbb{N}$.

Definition 2.1 A mapping $B: \mathbb{N}^{*} \rightarrow \mathbb{Z}_{+}$is called a branching function.

To each branching function corresponds a uniquely determined rooted tree $\mathbb{T}=(\mathbb{V}, \mathbb{A})$ with vertex set $\mathbb{V} \equiv \mathbb{V}^{*}(B) \subseteq \mathbb{N}^{*}$ and edge set $\mathbb{A}=\stackrel{\circ}{\mathbb{V}}$ defi ned as follows: $\mathbb{V}^{*}(B)=\cup_{n \in \mathbb{N}} \mathbb{V}^{n}(B)$ where $\mathbb{V}^{0}(B)=\{\emptyset\}=$ $\{$ root $\} \equiv\{\mathbf{0}\}$ and for $n \in \mathbb{N}$,

$$
\mathbb{V}^{n}(B)=\left\{\mathbf{v}=v_{1} \cdots v_{n}: v_{l} \in \mathbb{N}_{B\left(\left.\mathbf{v}\right|_{l-1}\right)}, \text { for } l=1, \ldots, n\right\} .
$$

The branching function is said to be without extinction if the corresponding tree has non-trivial boundary $\partial \mathbb{V}$. The edge set is the subset of unordered pairs of vertices $[\mathbf{u}, \mathbf{v}]=[\mathbf{v}, \mathbf{u}]$ such that either $\mathbf{v}=\hat{\mathbf{u}}$ or $\mathbf{u}=\hat{\mathbf{v}}$. Since every vertex has a unique ancestor, every edge is indexed by its outmost vertex, i.e. for every $\mathbf{v} \in \stackrel{\circ}{\mathbb{V}}$, the corresponding edge is $a(\mathbf{v})=[\hat{\mathbf{v}}, \mathbf{v}]$, showing thus that $\mathbb{A} \simeq \stackrel{\circ}{\mathbb{V}}$.

If $\mathbf{u}, \mathbf{v} \in \mathbb{V}$ and $\mathbf{u} \leq \mathbf{v}$ we defi ne the path $[\mathbf{u}, \mathbf{v}]$ as the collection of the $|\mathbf{v}|-|\mathbf{u}|$ edges $\left[\mathbf{u},\left.\mathbf{v}\right|_{|\mathbf{u}|+1}\right], \ldots,[\hat{\mathbf{v}}, \mathbf{v}]$, and if $\mathbf{u}=\emptyset$ then we simply denote by $[\mathbf{v}]$ the path $[\emptyset, \mathbf{v}]$ for every $\mathbf{v} \in \stackrel{\circ}{\mathbb{V}}$. In the sequel we shall consider only branching functions without extinction.

Definition 2.2 Let $\kappa_{n}=$ card $\mathbb{V}^{n}(B)$ denote the cardinality of the $n^{\text {th }}$ generation of the tree defi ned by the branching function without extinction $B$. We call lower growth rate of the tree

$$
\underline{\operatorname{gr}}(\mathbb{V})=\liminf _{n} \kappa_{n}^{1 / n}
$$

upper growth rate of the tree

$$
\overline{\operatorname{gr}}(\mathbb{V})=\underset{n}{\limsup } \kappa_{n}^{1 / n}
$$

and, if $\underline{\operatorname{gr}}(\mathbb{V})=\overline{\operatorname{gr}}(\mathbb{V})$, we call the common value growth rate

$$
\operatorname{gr}(\mathbb{V})=\lim _{n} \kappa_{n}^{1 / n}
$$

For $\mathbf{u}, \mathbf{v} \in \partial \mathbb{V}$, defi ne $\delta(\mathbf{u}, \mathbf{v})=\exp (-|\mathbf{u} \wedge \mathbf{v}|)$. It can be shown that $\delta$ is a distance on $\partial \mathbb{V}$. Moreover if $\|B\|_{\infty}=\sup _{\mathbf{v} \in \mathbb{V}} B(\mathbf{v})<\infty$ then the space $(\partial \mathbb{V}, \delta)$ is compact and we can defi ne its Hausdorff dimension $\operatorname{dim}_{H} \partial \mathbb{V}$ as usual (see [7] for instance).

Definition 2.3 For a tree $\mathbb{V}$ generated by a branching function $B$ with $\|B\|_{\infty}<\infty$, we defi ne its branching rate

$$
\operatorname{br}(\mathbb{V})=\exp \left(\operatorname{dim}_{H} \partial \mathbb{V}\right) .
$$

It is shown in $[21,16]$ that $\operatorname{br}(\mathbb{V})=\sup \left\{\lambda: \inf \sum_{v \in C} \lambda^{-|\mathbf{v}|}>0\right\}$ where the infi mum is evaluated over all cutsets $C$ of $\mathbb{V}$. We have in general that $\operatorname{br}(\mathbb{V}) \leq \underline{\operatorname{gr}}(\mathbb{V})$. 


\section{Matrix multiplicative cascades and the corresponding results}

Let $(\Omega, \mathcal{F}, \mathbb{P})$ be some abstract probability space which carries all the random variables that will be needed in the model. Let $(\mathbb{V}, \mathbb{A})$ be the rooted tree associated with a given branching function $B$. Let $G$ be the topological group $\mathrm{GL}(d, \mathbb{R}), \mathcal{G}$ its Borel $\sigma$-algebra and $\mu$ a probability on $(G, \mathcal{G})$. Denote by $\sigma_{\mu}=\operatorname{supp} \mu \subset$ $G$ the support of the measure $\mu$ and by $\Sigma_{\mu}$ the semi-group generated by $\sigma_{\mu}$. On $(\Omega, \mathcal{F}, \mathbb{P})$, defi ne an edgeindexed family of independent $G$-valued random variables $\left(\xi_{a}\right)_{a \in \mathbb{A}}$ identically distributed according to $\mu$, i.e.

$$
\mathbb{P}\left(\xi_{a} \in \mathrm{d} g\right)=\mu(\mathrm{d} g), \text { for all } a \in \mathbb{A} .
$$

For $\mathbf{u}, \mathbf{v} \in \mathbb{V}$ with $\mathbf{u} \leq \mathbf{v}$ defi ne

$$
\xi[\mathbf{u}, \mathbf{v}] \equiv \prod_{a \in[\mathbf{u}, \mathbf{v}]}^{\overleftarrow{ }} \xi_{a}
$$

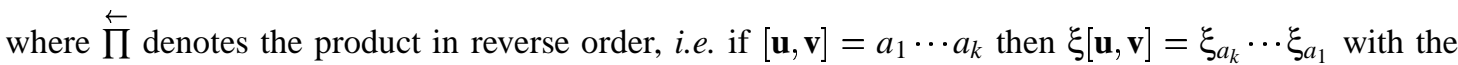
convention $\xi[\mathbf{v}, \mathbf{v}]=e$ where $e$ is the neutral element of $G$. We introduce the following $G$-valued random processes: the matrix-multiplicative cascade process

$$
\psi_{n}=\sum_{\mathbf{v} \in \mathbb{V}^{n}} \xi[\mathbf{v}], \quad n \in \mathbb{N}
$$

and the integrated matrix-multiplicative cascade process

$$
\zeta_{n}=\sum_{k=1}^{n} \psi_{k}, \quad n \in \mathbb{N}
$$

For a fi xed $\mathbf{v} \in \partial \mathbb{V}$ and all $n \in \mathbb{N}$

$$
X_{n} \equiv X_{n}(\mathbf{v})=\xi\left[\mathbf{v} \uparrow_{n}\right] .
$$

It is immediate to see (cf. [25]) that $\left(X_{n}\right)_{n \in \mathbb{N}}$ is a $G$-valued multiplicative Markov chain with stochastic kernel

$$
P\left(g, \mathrm{~d} g^{\prime}\right) \equiv \mathbb{P}\left(X_{n+1} \in \mathrm{d} g^{\prime} \mid X_{n}=g\right)=\mu \star \delta_{g}\left(\mathrm{~d} g^{\prime}\right), \quad n \in \mathbb{N},
$$

where for two measures $\mu, \mu^{\prime}$ on $(G, \mathcal{G})$ their convolution $\mu \star \mu^{\prime}$ is defi ned by its dual action on $L^{1}(G)$ via

$$
\left\langle\mu \star \mu^{\prime}, f\right\rangle \equiv \int_{G} f(g) \mu \star \mu^{\prime}(\mathrm{d} g)=\int_{G} \int_{G} f\left(g g^{\prime}\right) \mu(\mathrm{d} g) \mu^{\prime}\left(\mathrm{d} g^{\prime}\right),
$$

for all $f \in L^{1}(G)$. We equip $\operatorname{End}\left(\mathbb{R}^{d}\right) \equiv \mathrm{GL}(d, \mathbb{R})=G$ with the operator norm, denoted $\|\cdot\|$, stemming from the $l_{1}$ norm of the vector space $\mathbb{R}^{d}$.

In order to be able to apply the results of [9] to our special case, we require the following conditions on $\mu$ :

Condition 1 (Integrability): For all $s \in \mathbb{R}_{+}$,

$$
\int_{G}\|g\|^{s} \mu(\mathrm{d} g)<\infty
$$

Condition 2 (Strong irreducibility): We assume that the set $\Sigma_{\mu}$ is strongly irreducible, i.e. there is no fi nite $\Sigma_{\mu}$-invariant family of proper subspaces. 
Condition 3 (Strict positivity): We assume that $\sigma_{\mu} \subseteq \bar{G}_{+}$and that $\mu\left(\bar{G}_{+} \backslash G_{+}\right)=0$.

For $s \geq 0$, defi ne

$$
k(s)=\lim _{n}\left(\int_{G}\|g\|^{s} \mu^{\star n}(\mathrm{~d} g)\right)^{1 / n}
$$

(By virtue of theorem 1 of [9], under conditions 1-3 this limit exists in $\mathbb{R}_{+}$and defi nes a log-convex function. As a matter of fact, in [9] a weaker condition than 3, called proximality, is needed to prove this result.) We defi ne in the sequel the quantity $\lambda$, that turns out to be the main classifi cation parameter for the matrix multiplicative cascades model, by

$$
\lambda=\inf _{s \in[0,1]} k(s)
$$

(compare (3) with (1).)

We are now in the position to state our main results.

Theorem 3.1 Let $(\mathbb{V}, \mathbb{A})$ be some tree defined in terms of a given branching function $B$ and $\overline{\mathrm{gr}}(\mathbb{V})$ and $\lambda$ defined as in definition 2.2 and equation (3) respectively. Under the conditions 1, 2, and 3,

$$
\lambda \overline{\operatorname{gr}}(\mathbb{V})<1 \Rightarrow \zeta_{\infty, i j}<\infty \text { almost surely, for all } i, j=1, \ldots, d .
$$

Theorem 3.2 Let $\mathrm{br}(\mathbb{V})$ and $\lambda$ be the quantities introduced in definition 2.3 and equation 3 respectively and let $\chi \in \mathbb{R}^{d}$ be the vector having all its components equal to $1: \chi_{i}=1$, for all $i=1, \ldots, d$. Let $(\mathbb{V}, \mathbb{A})$ be some tree defined in terms of a given branching function B without extinction. Under the conditions 1 , 2, and 3',

$$
\lambda \operatorname{br}(\mathbb{V})>1 \Rightarrow Z_{\infty}:=\left(\chi, \zeta_{\infty} \chi\right)=\infty \text { almost surely. }
$$

Remark: Similarly to [16], there is a gap between Theorems 3.1 and 3.2, since in general the branching number need not be equal to the growth rate. However, this is not very important, because in most of the practical examples these quantities do coincide.

Remark: As mentioned above, the classifi cation parameter for this problem is $\lambda=\inf _{s \in[0,1]} k(s)$. This parameter is not explicitly computable in general since it involves the infi nite product of matrices. However for some particular cases this quantity can be computed explicitly as stated in the following proposition.

Proposition 3.3 Suppose that the measure $\mu$ is such that $g_{i j}<1 / d$ almost surely for all $i, j=1, \ldots, d$. Then $\lambda$ is the largest eigenvalue of the matrix $\mathbb{E} g$.

Remark: It is interesting to consider the chaos equation for the case of matrix-valued random variables and constant branching $b$ :

$$
Y \stackrel{\text { law }}{=} \sum_{j=1}^{b} Y_{j}^{\prime} \xi_{j}
$$

where $Y, Y_{j}^{\prime}, \xi_{j}$ are $G$-valued random variables, and $\xi_{j}$ (which are not necessarily independent) are distributed according to $\mu ; Y_{j}^{\prime}, j=1, \ldots, b$, are i.i.d. and have the same (unknown) law as $Y$. Analogously to [22] we can get that (at least in the case when $\xi_{1}$ satisfi es conditions $\left.1,2,3\right) \lambda d=1$ is a necessary condition for the existence of solution of (4). It is an open problem whether this condition is suffi cient.

Remark: The condition of independence of the random variables $\xi_{a}$ can be relaxed; what is important is

1. if $\xi_{a}$ and $\xi_{b}$ are not adjacent to the same vertex then they must be independent, and

2. the $\xi$ 's that belong to any path emanating from the root must be independent. 


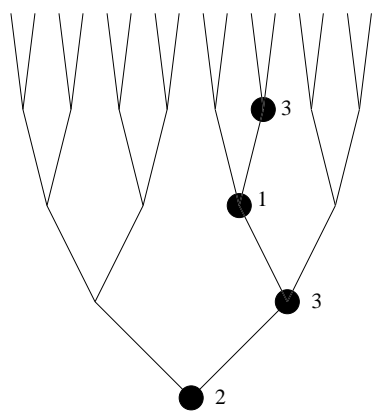

Fig. 1: A typical state of the bindweed model for $\mathcal{S}=\{1,2,3\}, \sigma=2313$, and $\mathbb{T}$ the binary tree.

\section{The bindweed model}

In this section we introduce a model describing an evolution of a random string in random environment on a tree (which is somewhat similar to the model studied in [4]) which we call the bindweed model. Then, we show that its classifi cation from the point of view of positive recurrence can be obtained by using theorems 3.1 and 3.2.

Let $S=\{1, \ldots, d\}$ be a fi nite alphabet and denote, in accordance with the notations introduced in Section $2, \mathcal{S}^{n+1}=\left\{\sigma=\sigma_{0} \cdots \sigma_{n}: \sigma_{i} \in \mathcal{S}\right\}$ the set of words of length $n+1$ composed from the symbols of the alphabet $\mathcal{S}, \mathcal{S}^{0}$ the set containing only the empty word and $\mathcal{S}^{*}$ the set of words of arbitrary length. Suppose that a branching function $B$ is given on $\mathbb{N}^{*}$ and denote $\mathbb{V}^{n} \equiv \mathbb{V}^{n}(B)$ the corresponding generations of the tree determined by $B$. Therefore, the rooted tree $\mathbb{T}=(\mathbb{V}, \mathbb{A})$ is uniquely defi ned.

Now we are going to construct a continuous-time Markov chain with state space $\mathfrak{S}$, defi ned by

$$
\mathfrak{S}=\{\hat{\emptyset}\} \cup \bigcup_{n=1}\left(\mathbb{V}^{n} \times \mathcal{S}^{n+1}\right)
$$

where $\hat{\emptyset}$ is a special state to be defi ned later. In fact, what happens is the following: we place a word $\sigma=\sigma_{0} \ldots \sigma_{n}$ on the tree $\mathbb{T}$ in such a way that the $0^{\text {th }}$ symbol of the word is placed on the root $\mathbf{0}$, for any $i=1, \ldots, n$ the $i^{\text {th }}$ symbol of the word is placed somewhere in $\mathbb{V}^{i}$, and, if the $i^{\text {th }}$ symbol $\sigma_{i}$ is placed on vertex $\mathbf{u}$, and $\sigma_{i+1}$ on $\mathbf{v}$, then $\mathbf{u}<\mathbf{v}$ and $[\mathbf{u}, \mathbf{v}] \in \mathbb{A}$ (see fi gure 1 ). The state $\hat{\emptyset}$ means that nothing is placed on the tree.

Now, let us defi ne the dynamics of the bindweed model. Suppose that for any $a \in \mathbb{A}$ two collections of positive numbers $\left(v_{y z}(a), y, z \in S\right),\left(\mu_{y}(a), y \in S\right)$ are given. If the bindweed model is in the state $(\mathbf{u}, \sigma)$, where $\sigma=\sigma_{0} \ldots \sigma_{n-1} y, \mathbf{u} \in \mathbb{V}^{n}$, then

- for $n \geq 0$ it jumps to the state $\left(\mathbf{v}, \sigma_{0} \ldots \sigma_{n-1} y z\right)$ with rate $v_{y z}(a(\mathbf{v}))$, for all $\mathbf{v} \in \mathbb{V}: \mathbf{u}=\hat{\mathbf{v}}$;

- for $n \geq 1$ it jumps to the state $\left(\hat{\mathbf{u}}, \sigma_{0} \ldots \sigma_{n-1}\right)$ with rate $\mu_{y}(a(\mathbf{u}))$.

For any $\sigma_{0} \in \mathcal{S}$ the transitions $\hat{\emptyset} \rightarrow\left(\mathbf{0}, \sigma_{0}\right)$ and $\left(\mathbf{0}, \sigma_{0}\right) \rightarrow \hat{\emptyset}$ occur with rate 1 . Thus, we have defi ned a continuous-time Markov chain with state space $\mathfrak{S}$.

Let us describe now how to choose the transition rates. Let $\rho$ be any probability measure on $\mathbb{R}_{+}^{d^{2}+d}$. Suppose that for any $a \in \mathbb{A}$ the vector $\Xi(a)=\left(v_{y z}(a), y, z \in \mathcal{S}, \mu_{y}, y \in \mathcal{S}\right)$ is random, having distribution 
$\rho$, and $(\Xi(a), a \in \mathbb{A})$ are independent and identically distributed. Fix a realization of that collection of random vectors and consider the bindweed model with the transition rates ruled by that realization. So, the model that we constructed is a continuous-time Markov chain in a quenched random environment.

Now, we are interested in obtaining a classifi cation of this Markov chain with respect to positive recurrence. For $(\mathbf{v}, \sigma) \in \mathfrak{S}$ denote by $\pi(\mathbf{v}, \sigma)$ the stationary measure. For any $a \in \mathbb{A}$ let $\xi_{a}$ be a $d \times d$ matrix whose matrix elements are defi ned in the following way: $\xi_{\mathfrak{s}, x y}=v_{x y}(a) / \mu_{y}(a), x, y \in S$. It is not diffi cult to see that we have a reversible Markov chain, so it is clear that $\pi(\hat{\emptyset})=\pi(\mathbf{0}, x)$, for all $x \in \mathcal{S}$, and, for any $\mathbf{v} \in \mathbb{V}^{n}, n \geq 1$, and $x, y, \sigma_{0}, \ldots, \sigma_{n-2} \in \mathcal{S}$, we can formally write

$$
\begin{aligned}
\pi\left(\mathbf{v}, \sigma_{0} \ldots \sigma_{n-2} x y\right) & =\frac{v_{x y}(a(\mathbf{v}))}{\mu_{y}(a(\mathbf{v}))} \pi\left(\hat{\mathbf{v}}, \sigma_{0} \ldots \sigma_{n-2} x\right) \\
& =\xi_{a(\mathbf{v}), x y} \pi\left(\hat{\mathbf{v}}, \sigma_{0} \ldots \sigma_{n-2} x\right)
\end{aligned}
$$

Then it is shown in [23] that

$$
\sum_{\substack{\mathbf{v} \in \mathbb{V}^{n} \\ \sigma \in S^{n}}} \pi(\mathbf{v}, \sigma)=\pi(\hat{\emptyset})\left(\chi, \sum_{\mathbf{v} \in \mathbb{V}^{n}} \xi[\mathbf{v}] \chi\right)
$$

where $\chi$ is the vector of order $d$ with all its coordinates equal to 1 . Thus $\sum_{(\mathbf{v}, \sigma) \in \mathfrak{S}} \pi(\mathbf{v}, \sigma)$ is fi nite if and only if $Z_{\infty}$ is fi nite. Thus, theorems 3.1 and 3.2 allow us to obtain the classifi cation of the bindweed model in random environment from the point of view of positive recurrence, in the following way:

Proposition 4.1 Suppose that the distribution of the random matrix $\xi_{a}$ is such that the Conditions 1, 2, and 3 are satisfied. Let $\lambda$ be the quantity defined as in Section 3. Then

- if $\lambda \overline{\mathrm{gr}}(\mathbb{V})<1$, then the bindweed model is positive recurrent;

- if $\lambda \operatorname{br}(\mathbb{V})>1$, then the bindweed model is not positive recurrent.

\section{Open problems and further developments}

We demonstrated a close relationship between matrix multiplicative cascades and random walks in random environment on multiplexed trees. In particular it is proved in [23] that both systems are classifi ed by the same parameter. However, the critical region remains out of reach for the moment. Firstly it is not known whether, for suffi ciently regular trees so that $\operatorname{gr}(\mathbb{V})=\operatorname{br}(\mathbb{V})=b$, the walk is null recurrent for $\lambda b=1$ or some additional condition is needed on $\mu$ as is the case for scalar multiplicative chaos [14] and for random walk [16]. Returning to the general tree where $\overline{g r}(\mathbb{V}) \neq \operatorname{br}(\mathbb{V})$, we obtain a gap in the space of classifying parameters. It is however conjectured in [17] that in general, the set of critical values is of zero Lebesgue measure for string problems. We expect the same phenomenon to occur here. Nevertheless, whether the critical value of the parameter is $\operatorname{br}(\mathbb{V})$ or $\overline{g r}(\mathbb{V})$ or some intermediate value is unknown for the moment.

An important step toward understanding these problem should be made if conditions for the existence of non-trivial fi xed points of the functional equation (4) were obtained. This remains for the moment an open problem although under investigation. 


\section{References}

[1] M. Campanino and D. Petritis. On the physical relevance of random walks: an example of random walks on randomly oriented lattices, in "Random walks ans geometry", V. Kaimanovich and W. Woess eds., in press, (2003).

[2] M. Campanino and D. Petritis. Random walks on randomly oriented lattices, preprint 2001, eprint arXiv:math.PR/0111305, to appear in Markov Proc. Rel. Fields (2003).

[3] P. Collet and F. Koukiou. Large deviations for multiplicative chaos. Commun. Math. Phys., 147:329342, 1992.

[4] F. Comets, M. Menshikov, and S. Popov. Lyapunov functions for random walks and strings in random environment. Ann. Probab., 26:1433-1445, 1998.

[5] F. Comets and S.Yu. Popov. Limit law for transition probabilities and moderate deviations for sinai's random walk in random environment, To appear in: Probab. Theory Relat. Fields, 2003.

[6] R. Durrett and Th. Liggett. Fixed points of smoothing transformation. Z. Wahrscheinlichkeitstheorie verw. Gebiete, 64:275-301, 1983.

[7] K. J. Falconer. The geometry of fractal sets. Cambridge University Press, Cambridge, 1986.

[8] Ph. Flajolet and R. Sedgwick. Analytic combinatorics: functional equations, rational and algebraic functions, Research report 4103 INRIA, 2001.

[9] Yves Guivarc'h and Émile Le Page. Simplicité de spectres de Lyapunov et propriété d'isolation spectrale pour une famille d'opérateurs sur l'espace projectif, to appear in Random walks and geometry, V. Kaimanovich and W. Woess, eds.

[10] J.-P. Kahane and J. Peyrière. Sur certaines martingales de Benoît Mandelbrot. Adv. Math., 22:131$145,1976$.

[11] H. Kesten, M.V. Kozlov, and F. Spitzer. A limit law for random walk in a random environment. Compositio Math., 30:145-168, 1975.

[12] Harry Kesten. The limit distribution of Sinaǐ's random walk in random environment. Phys. A, 138(1-2):299-309, 1986.

[13] A. Kumjian, D. Pask, I. Raeburn, and J. Renault. Graphs, groupoids, and Cuntz-Krieger algebras. J. Funct. Anal., 144(2):505-541, 1997.

[14] Q. Liu. Sur une équation fonctionnelle et ses applications: une extension du théorème de KestenStigun concernant les processus de branchement. Ann. Appl. Probab., 29:353-373, 1997.

[15] Quansheng Liu and Alain Rouault. Limit theorems for Mandelbrot's multiplicative cascades. Ann. Appl. Probab., 10(1):218-239, 2000.

[16] R. Lyons and R. Pemantle. Random walk in a random environment and fi rst passage percolation on trees. Ann. Probab., 20:125-136, 1991. 
[17] V. A. Malyshev. Interacting strings of symbols. Uspekhi Mat. Nauk, 52(2(314)):59-86, 1997.

[18] V. A. Malyshev. Random grammars. Uspekhi Mat. Nauk, 53(2(320)):107-134, 1998.

[19] V. A. Malyshev. Stochastic evolution via graph grammars, Research report 3380 INRIA, 2001.

[20] Benoit Mandelbrot. Multiplications aléatoires itérées et distributions invariantes par moyenne pondérée aléatoire: quelques extensions. C. R. Acad. Sci. Paris Sér. A, 278:355-358, 1974.

[21] R. D. Mauldin, S. Graf, and S. C. Williams. Exact Hausdorff dimension in random recursive constructions. Proc. Nat. Acad. Sci. U.S.A., 84(12):3959-3961, 1987.

[22] M. Menshikov and D. Petritis. Random walks in random environment on trees and multiplicative chaos, Mathematics and computer science II, B. Chauvin, Ph. Flajolet, D. Gardy, A. Mokkadem, eds, pp. 415-422, Birkhäuser, Basel (2002).

[23] M. Menshikov, D. Petritis, and S. Popov. Matrix multiplicative chaos and bindeweeds, preprint 03-05 IRMAR 2003.

[24] D. Petritis. Directed graphs driven grammars, in preparation 2003.

[25] D. Revuz. Markov chains. North-Holland Publishing Co., Amsterdam, second edition, 1984.

[26] Ya. G. Sină̌. The limit behavior of a one-dimensional random walk in a random environment. Teor. Veroyatnost. i Primenen., 27(2):247-258, 1982.

[27] F. Solomon. Random walk in a random environment. Ann. Probab., 3:1-31, 1975. 
